\title{
ARistotele E IL PROBLEMA DELLA SOGGETTIVITÀ
}

\author{
Marco Sgarbi ${ }^{1}$
}

\begin{abstract}
RIASSUNTO: Gli storici della filosofia solitamente attribuiscono ai pensatori moderni l'invenzioni di concetto "soggetto", "oggetto" e "soggettività" e li concepiscono in direttamente in contrasto con la filosofia scolastica e aristotelica. Questo articolo suggerisce la presenza di una proto-teoria della soggettività in Aristotele. La teoria aristotelica della soggettività è fondata principalmente su quattro dottrine. In primo luogo, si può riscontrare nell'epistemologia aristotelica la presenza di una "prospettiva" e di un "punto di vista". Il secondo aspetto significativo è il concetto di determinazione dell'oggetto della conoscenza, che nella filosofia aristotelica è rappresentato dalla nozione di "prosthesis". La terza dottrina si fonda sulla distinzione fra ciò che è più conoscibile per noi (gnorimoteron emin) e ciò che è più conoscibile per natura (gnorimoteron te physei). Lultima dottrina riguarda la seconda natura del soggetto e gli abiti della conoscenza acquisiti attraverso l'esperienza, i quali spiegano perché i soggetti abbiano conoscenze diverse degli stessi oggetti secondo le loro precedenti esperienze.
\end{abstract}

PAROLE-CHIAVI: Aristotele. Soggettività. Determinazione. Prospettiva.

Concetti filosofici chiave come "soggetto", "oggetto", "soggettivo", "oggettivo" sono sempre stati al centro dell'interesse degli storici della filosofia, soprattutto di quelli che si sono occupati del pensiero moderno ${ }^{2}$. Per lungo tempo si è pensato, sulla scia delle riflessioni di Georg Wilhelm Friedrich Hegel e Martin Heidegger, che i concetti di "soggetto" con il suo correlato di "oggetto" fossero un tipico prodotto della filosofia moderna e in particolar modo del pensiero di René Descartes. Più recentemente, grazie a rinnovate e più accurate ricerche di storia

\footnotetext{
${ }^{1}$ Dipartimento di Filosofia della Università degli Studi di Verona.

${ }^{2}$ Esempio sono le voci "Objekt", "Subjekt", "Subjekt/Obekt; subjektiv/objektiv" dell'Historisches Wörterbuch der Philosophie che sono incentrate prevalentemente sulla storia del concetto nella modernità, cf. KOBUSCH, T. "Objekt", in: RITTER, J. (cur.). Historisches Wörterbuch der Philosophie. Basel: Schwabe, 1984, VI, coll. 1026-1052; KIBLE, B.; STOLZENBERG, J.; TRAPPE, T; DREISHOLTKAMP, U. "Subjekt", in: RITTER, J. (cur.). Historisches Wörterbuch der Philosophie. Basel: Schwabe, 1998, X, coll. 373-400;. KARSKENS, K.; KNEBEL, S. K.; ONNASCH, E.-O. "Subjekt/Objekt; subjektiv/objektiv, in: RITTER, J. (cur.). Historisches Wörterbuch der Philosophie. Basel: Schwabe, 1998, X, coll. 401-433.
} 
dei concetti e delle idee, la storiografia filosofica, in primis Lawrence Dewan, Alain De Libera e Olivier Boulnois ${ }^{3}$, ha stabilito che i concetti di "soggetto" e "oggetto" sono un'invenzione del pensiero medievale.

Più tarda è l'origine del concetto di cui si vuole trattare in questo articolo, cioè quello di "soggettività". Il concetto di "soggettività" come sostantivizzazione del concetto di "soggettivo" entra nel comune lessico filosofico solo verso la fine del XVIII secolo con la filosofia kantiana ${ }^{4}$. Le ricerche storiografiche, tuttavia, hanno dimostrato che né Kant e né i suoi seguaci avevano di essa un concetto ben definito 5 . Il punto di svolta nella storia del concetto di "soggettività" è rappresentato dallo scritto Glauben und Wissen del 1802 di Hegel, molto significativamente sottotitolato die Reflexionsphilosophie der Subjectivität. Questo scritto rappresenta

[...] la prima esplicita teoria filosofica della soggettività, in quanto qui il problema della soggettività viene per la prima volta indagato sistematicamente sotto differenti dimensioni e aspetti e viene posto un nuovo concetto come linea guida della ricerca. ${ }^{6}$.

Dalla piccola opera di Hegel si possono evincere almeno cinque idee basi che aiutano a definire una teoria della soggettività.

La prima idea afferma che la soggettività offre un punto di vista particolare rispetto all'oggetto della conoscenza, cioè non considera l'oggetto nella sua totalità. La seconda idea, connessa alla prima, è quella della determinazione, cioè che l'oggetto della conoscenza viene determinato in uno specifico modo secondo la prospettiva del soggetto. A questa seconda idea si riconduce anche la terza idea per la quale gli oggetti della conoscenza hanno un significato solo per il soggetto conoscente e non sono portatori di significati in sé: è la mente che significa le cose del mondo. Il punto di vista, la determinazione epistemica e la capacità di dare significati (o capacità ermeneutica) caratterizzano le idee di riflessione, secondo la quale ciò che è esterno deve diventare interno, e di egoità (o ispeità), cioè dell'esistenza di un punto fisso centro di tutte queste operazioni.

3 Cf. DEWAN, L. Obiectum. Notes on the Invention of a Word.Archives d'histoire doctrinale et littéraire du moyen âge, 48, p. 37-96, 1981; DE LIBERA, A. Archéologie du sujet. Paris: Vrin, 2007; BOULNOIS, O. (cur.). Généalogies du sujet. De Saint Anselme à Malebranche. Paris: Vrin, 2007; DE LIBERA, A. When did the Modern Subject Emerge? American Catholic Philosophical Quarterly, 82, p. 181-220, 2008.

${ }^{4}$ Cf. CLAIRMONT, H. "Subjektivität", in J. RITTER, J. (cur.). Historisches Wörterbuch der Philosophie. Basel: Schwabe, 1998, X, coll. 458-464;

5 Cf. HOMANN, K. Zum Begriff "Subjektivität" bis 1802. Archiv für Begriffsgeschichte, 11, p. 184205, 1967.

${ }^{6}$ IVI, p. 197. 
1) Punto di vista, 2) determinazione, 3) capacità emerneutica, 4) riflessione ed 5) egoità sono perciò sono le idee base per la fondazione di una filosofia della soggettività che secondo Hegel avrebbe in Kant il suo primo grande teorizzatore. Nel presente contributo si vuol dimostrare, invece, che una proto-teoria della soggettività, così come l'ha concepita Hegel, è stata elaborata per la prima volta da Aristotele.

La presenza dell'idea di una prospettiva, di un punto di vista della conoscenza è sicuramente uno degli indici più importanti per valutare la presenza di una teoria della soggettività in qualsiasi autore. Lidea di una prospettiva, o di un punto di vista della conoscenza, non deve essere confusa con la possibilità degli uomini di conoscere diversamente i vari oggetti in tempi e luoghi diversi, bensì è quel fatto gnoseologico per il quale i vari soggetti conoscono costitutivamente in modo differente i medesimi oggetti nello stesso tempo e nello stesso spazio. Avere un punto di vista non significa perciò avere un'opinione differente rispetto ad un oggetto della conoscenza, ma avere proprio un'altra immagine nella mente dell'oggetto conosciuto. In questo senso il fatto che l'immagine della conoscenza corrisponda effettivamente o realmente all'oggetto conosciuto, cioè che si possa affermare la verità o la falsità su di esso, è del tutto irrilevante. Non si sta parlando di una logica apofantica o epistemica, ma piuttosto di una logica ermeneutica, cioè quella logica attraverso la quale è possibile attribuire diversi significati all'oggetto della conoscenza.

Caratterizzare la logica e la gnoseologia aristotelica come una logica ermeneutica significa riconoscere una certa attività originaria dell'uomo nella conoscenza che le più comuni interpretazioni aristoteliche, supportate da quanto lo stesso Stagirita afferma nei libri del De anima, negano. Infatti, la maggior parte degli interpreti di Aristotele affermano che le cose sensibili (ta aistheta) affettano l'anima che le riceve passivamente senza alcuna attività della mente.

In realtà ci sono diversi luoghi nei quali Aristotele, insieme ad altre numerose precisazioni di metodo, sembra parlare di una differente costituiva possibilità di considerare le cose. Il più importante passo è sicuramente quello di Ethica nicomachea I.7, 1098 a 25-35 nel quale Aristotele afferma che non è necessario "[...] cercare in eguale misura l'esattezza in tutti gli argomenti, ma in ciascun argomento conformemente alla cosa considerata e tanto quanto è proprio dell'indagine". Questo prima parte del passo richiama un altro importante luogo aristotelico di Metaphysica II.3, 995 a 15-30, nel quale si afferma che è necessario essere istruiti sul "come" (pos) ciascuna scienza va trattata, non si può infatti ricercare insieme la scienza e il tropos epistemes. Il concetto di tropos epistemes è senz'altro fondamentale nella teoria epistemologica aristotelica. Tropos significa il più delle volte modo, maniera, metodo, ma anche direzione, verso, disposizione, 
atteggiamento, e in questo specifico caso di colui che conosce verso ciò che è conosciuto. Si può ben dire quindi che il tropos epistemes indica per Aristotele il punto di vista, la prospettiva che si adotta o che si dovrebbe adottare nelle varie indagini conoscitive. Ad esempio, non deve essere adottato un tropos epistemes troppo rigoroso per gli oggetti che hanno la materia, come invece si dovrebbe fare per gli oggetti puramente matematici. Il passo di Ethica nicomachea I.7 è particolarmente interessante anche per la presenza di almeno altri tre termini assai significativi per caratterizzare la teoria della soggettività di Aristotele. Sono i termini hypokeimenon, hyle e methodos. Il termine greco hypokeimenon significa ciò che sta sotto, ciò che è gettato sotto ed è tradotto con il latino subjectum dal quale deriva il termine comune a tutte le lingue vernacolari di "soggetto", "subject", "Subjekt", "sujet", "subjecto" etc... Il termine hyle viene tradotto solitamente con materia e viene in questo passo concepito in riferimento all'oggetto della conoscenza: cioè la materia della conoscenza. Si tratta di un'annotazione assai importante perché per la prima rivolta ricorre il concetto di una "materia" vera e propria della conoscenza in opposizione alla forma della conoscenza o methodos che tanta fortuna avrà a partire dalla logica del Cinquecento. Il termine methodos, infatti, significa il corrente termine "metodo", ma inteso come via, modo o forma della trattazione. Il fatto che si tratti di una forma della conoscenza, cioè di una prospettiva particolare di colui che conosce sull'oggetto conosciuto è testimoniato dalle righe successive del passo di Ethica nicomachea I.7. Qui Aristotele, riprendendo il discorso fatto in precedenza sulla diversità delle forme di considerazione della materia conosciuta, afferma che "[...] infatti, un costruttore e un geometra osservano diversamente un angolo retto: giacché il primo lo osserva per quanto gli è utile per ciò che deve produrre, mentre il secondo, invece, osserva il genere e la differenza

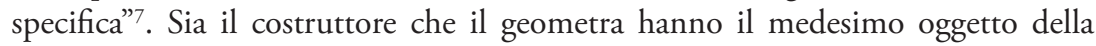
conoscenza, l'angolo retto, ma lo esaminano, lo considerano diversamente, cioè con "intenzioni" differenti, ed è proprio questa l'idea che sancisce la differenza dei punti di vista rispetto a qualità specifiche di colui che conosce ${ }^{8}$. Di che tipo siano queste differenti qualità del conoscente lo si vedrà poi in seguito. Basti ora ad affermare che per ogni materia oggetto alla conoscenza c'è un tropos epistemes differente che corrisponde al punto di vista di colui che conosce e questa idea esprime una proto-teoria della soggettività.

Il secondo aspetto rilevante per la fondazione di una teoria della soggettività è quello della determinazione della materia della conoscenza. Riprendendo la

\footnotetext{
${ }^{7}$ ARISTOTELE, Ethica nicomachea, I.7, 1098 a 25-35.

${ }^{8}$ Sull'interpretazione di questo passo nell'aristotelismo medievale e rinascimentale e il suo influsso sulla genesi della soggettività cf. POZZO, R. Res considerata and modus considerandi rem: Averroes, Aquinas, Jacopo Zabarella, and Cornelius Martini on Reduplication. Medioevo, 24, p. 251-267, 1998.
} 
formulazione spinoziana - omnis determinatio est negatio - una determinazione è sempre una delimitazione di ciò che in origine non aveva un limite. In termini logici significa che ad un soggetto viene aggiunta una nota che prima non gli apparteneva per caratterizzarlo in modo più specifico.

In Aristotele questo concetto ritorna spesso sia nel corpus logico che in quello metafisico sotto la forma del concetto di prosthesis che significa "aggiunta", "addizione", ma anche appunto "determinazione". Il termine prosthesis significa letteralmente "porre davanti" e richiama spesso la concettualità legata all'antikeimenon, cioè a ciò che è gettato di fronte come qualcosa di indeterminato, da cui deriva il latino "objectum", base di termini quali "oggetto", "objecto", "object", "Objekt" etc...

Fra i molti passi, uno dei più significativi è sicuramente quello di Analytica posteriora I.27: “[...] per determinazione dico per esempio: l'unità è una sostanza senza posizione (athetos), mentre il punto è una realtà con posizione (thetos); quest'ultima è per determinazione" ${ }^{10}$. L'unità è in sé una realtà indeterminata, cioè che non pone; il punto, al contrario, è qualcosa di determinato in quanto posto. La mancanza di determinazione porta ad una considerazione aplos (simpliciter) della cosa conosciuta, mentre evidentemente la determinazione conduce ad una conoscenza della cosa secundum quid (pros ti), il quale altro non è che una posizione determinata tenuta da colui che conosce. La determinazione specifica così ciò che altrimenti sarebbe indeterminato ed in definitiva come tale non conoscibile. Il genere di determinazione che ha in mente in questo contesto Aristotele è proprio quella della definizione alla quale si aggiunge una nota caratteristica. In questo senso si esprime Aristotele in Topica II.11, 115a 25-35 e in Topica III.6, 119a 2025. In Metaphysica VII.4, 1029 b 29-33 Aristotele afferma che ciò che non è per sé (to ou kath'auto) si dice in due sensi uno dei quali è secondo la determinazione: "[...] ciò che si vuole definire viene aggiunto ad altro come predicato, come per esempio se volendo definire l'essenza del bianco, si fornisse la definizione di uomo bianco". Tale procedimento è poi per Aristotele quello tipico della definizione delle categorie differenti della sostanza: la loro definizione è solo per determinazione (cioè attraverso una relazione). Infatti, come accade "[...] nel caso della qualità e del dispari: non c'è il dispari senza il numero, come non c'è femmina senza l'animale". La determinazione per Aristotele è una concretizzazione di ciò che

${ }^{9}$ Cf. POZZO, R. Soggetto e determinazione nella tradizione aristotelica. In: DE BELLIS, E. (cur.). Aristotle and the Aristotelian Tradition. Innovative Contexts for Cultural Tourism. PROCEEDINGS OF THE INTERNATIONAL CONFERENCE. Lecce - June 12, 13, 14, 2008, Rubbettino, Soveria Mannelli 2008, p. 305-310.

${ }^{10}$ ARISTOTELE, Analytica posteriora, I.27, 87 a 31-37. 
altrimenti sarebbe aplos, ma che proprio perciò non sarebbe un possibile oggetto della conoscenza.

Riveste un ruolo centrale nella teoria logico-epistemologica aristotelica il problema se le cose in sé, indipendentemente da chi le conosce, abbiano o no un significato e si ci sia un parallelismo fra l'ordine mentale e l'ordine reale. Questa domanda coinvolge uno degli aspetti metodologici più importanti della riflessione filosofica aristotelica, cioè la distinzione fra ciò che è più noto per l'uomo (gnorimoteron emin) e più noto per sé (gnorimoteron te physei) ${ }^{11}$. Nel corpus aristotelico la distinzione occorre per la prima volta in Topica VI.4, 141 b 3-22 proprio in merito al problema della definizione. La definizione, secondo Aristotele, è composta o da elementi meno noti a noi o da elementi meno noti in senso assoluto. Più noto per sé, in senso assoluto, è ciò che è anteriore rispetto a ciò che è posteriore. Gli elementi semplici sono più noti per sé degli elementi determinati, tuttavia, accade che gli uomini conoscano prima ciò che più determinato e poi ciò che è più semplice (aplos), ad esempio la sillaba rispetto la lettera. Questo è possibile perché l'uomo è più vicino a ciò che proviene dai sensi e solo la mente più acuta può cogliere gli elementi più noti in senso assoluto (aplos): le cose più universali sono in generale quelle più lontane dall'uomo, mentre quelle singolari sono quelle più vicine ${ }^{12}$. Per gli uomini perciò saranno più note le cose sensibili, mentre una volta acquisita molta esperienza saranno più note le cose intelligibili ${ }^{13}$. Si tratta di una mera differenza gnoseologica, infatti, per Aristotele, ciò che è più noto in senso assoluto non è qualcosa di ontologicamente differente da ciò che è più noto per l'uomo, è solo conosciuto in modo meno determinato. In questo modo Aristotele evita qualsiasi accusa di possibile idealismo e apre le porte ad una vera teoria della soggettività.

La distinzione ricorre nella sua formulazione più nota proprio nel capitolo introduttivo della Physica, nel quale Aristotele tratta i principî della scienza della natura. Conoscere, ribadisce Aristotele, significa avere conoscenza delle cause prime, dei principî primi e degli elementi originari. Aristotele afferma che "[...] è naturale che si proceda da ciò che è più noto e chiaro per noi verso quello che è più chiaro e noto per natura; infatti non sono la medesima cosa il conoscibile per noi e il conoscibile in senso assoluto" ${ }^{14}$. Il procedimento da adottare-afferma

${ }^{11}$ Cf. Mansion, S. "Plus connu en soi", "plus connu pour nous". Une distinction epistemologique importante chez Aristote. Pensamiento, 35, p. 161-170, 1979; BOLTON, R. Aristotle's Method in Natural Science: Physics I. In: JUDSON, L. (cur.). Aristotle’s Physics: A Collection of Essays. Oxford: Clarendon Press, 1991, p. 1-29.

${ }^{12}$ ARISTOTELE, Analytica posteriora, I.2, 72 a 1-5.

${ }^{13}$ ARISTOTELE, Topica, VI.4, 142 a 3-4.

${ }^{14}$ ARISTOTELE, Physica, I.1, 184 a 16-18. 
Aristotele-è quello di partire da ciò che è meno chiaro per natura ma più chiaro per noi al fine di conoscere ciò che è più chiaro per natura. La ricerca di ciò che è più chiaro e conoscibile per natura corrisponde in Aristotele alla ricerca della verità. Ricercare la verità, scrive Aristotele in Metaphysica II.1, non è facile perché ciò che è più noto per natura è per noi più oscuro. Gli uomini si comportano con la verità come gli occhi delle nottole nei confronti della luce del sole; di fronte alle cose più evidenti per natura essi si confondono ${ }^{15}$. Ciò che è più noto per noi è ciò che deriva dalla sensazione e ciò che è per noi più chiaro ed evidente sono le cose nel loro insieme. Aristotele sta parlando dell'induzione imperfetta (epagoge) discussa in Analytica posteriora II.19, attraverso la quale dopo molteplici sensazioni, dopo molte esperienze e dopo la ritenzione delle immagini nella memoria si ottiene un concetto generale (katholou $)^{16}$.

Tutta questa teoria si basa però sul presupposto che quando conosciamo qualche cosa, la conosciamo per mezzo di qualcosa d'altro, cioè ci si interroga sulla cosa ignota attraverso qualcosa che è già noto. Non si può perciò conoscere immediatamente una cosa da se stessa per Aristotele, escludendo così ogni possibile dottrina intuizionistica, in stile Platone, che minerebbe alle basi la possibilità di una teoria della soggettività. Si deve sempre presupporre qualcosa che è già conosciuto, una pre-conoscenza, un pre-giudizio, una pre-comprensione, cioè che i medievali chiamavano conoscenza ex praecognitis et praeconcessis: "[...] ogni insegnamento (didaskalia) e ogni apprendimento discorsivo (mathesis dianoetike) nasce da una conoscenza preesistente" ${ }^{17}$.

La distinzione fra ciò che è primo per sé e ciò che è primo per l'uomo stabilisce due vie per la conoscenza della cosa, indipendentemente dal fatto che si possano attuare entrambe: 1) quella con presupposti, o soggettiva; e 2) quella nella quale i presupposti sono dimostrati, o oggettiva. Ciò che si conosce è sempre lo stesso; si tratta invece di due metodi d'indagine differenti e quindi prospettive e punti di vista diversi. A questa analisi si potrebbe obiettare che il sapere non è per Aristotele nulla che possa essere ricondotto a una sfera soggettiva della coscienza perché il concetto di sapere, come cognitio rei per causam, non si riferisce tanto all'agente, ma al suo contenuto o in modo più specifico alla causa formale ${ }^{18}$. A questa tesi si può replicare che per Aristotele le cause in sé non appartengono

\footnotetext{
${ }^{15}$ ARISTOTELE, Metaphysica, II.1, 993 b 7-11.

${ }^{16}$ ARISTOTELE, Analytica Posteriora, II.19, 99 b 35-100 b 5.

${ }^{17}$ ARISTOTELE, Analyticia posterioria, I.1, 71 a 1.

18 WIELAND, W. Die aristotelische Physik. Untersuchungen über die Grundlegung der Naturwissenschaft und die sprachlichen Bedingungen der Prinzipienforschung bei Aristoteles. Göttingen: Vandenhoeck \& Ruprecht, 1962, p. 69-70.
} 
realmente alle cose, ma sono strumenti euristici della mente per indagare le cose del mondo ${ }^{19}$.

Inoltre, l'obiettivo di Aristotele in questi passi è proprio quello di sottolineare il ruolo centrale del conoscente rispetto alla cosa conosciuta spiegando le due diverse modalità di accesso alla realtà. Non sto suggerendo che in Aristotele sia presente una sfera della coscienza, tuttavia, il fatto che la medesima cosa possa essere conosciuta in due modi differenti non può riguardare ciò che si conosce, quanto piuttosto il punto di vista del conoscente, cioè appunto la sua soggettività. Se non ci fosse una sfera della soggettività non sarebbe possibile indagare in modi differenti le cose. Ci sarebbe solo una conoscenza univoca ed universale della cosa che negherebbe l'esperienza quale fattore determinate nella formazione dell'abito della scienza nei vari individui. Si tratta del problema che nella tradizione aristotelica sarà definito "seconda natura" e che rispecchia il tema della riflessione.

Il tema della "seconda natura" in relazione alla soggettività, ripreso recentemente dalla filosofia della mente di John $\mathrm{H}$. McDowell ${ }^{20}$, coinvolge direttamente la questione dell'habitus in Aristotele. Il luogo più importante e famoso in cui la teoria aristotelica dell'habitus è sviluppata è nel libro sesto dell'Ethica nicomachea, nel quale Aristotele individua le cinque virtù dianoetiche: 1) arte; 2) saggezza; 3) intelletto; 4) scienza; 5) sapienza ${ }^{21}$. Destano particolare interesse l'abito dell'intelletto e della scienza. Per scienza Aristotele intende quella conoscenza che non può essere diversamente da ciò che $\mathrm{e}^{22}$. L'oggetto della scienza è perciò necessario. La conoscenza di questo oggetto è tuttavia possibile solo attraverso una dimostrazione ben argomentata e fondata su principî noti ${ }^{23}$. L'abito dell'intelligenza è proprio quello che fornisce la conoscenza dei principî sui quali si basa l'argomentazione della conoscenza scientifica ${ }^{24}$.

19 IVI, p. 202-230. Di questa opinione è anche Enrico Berti, sebbene egli aggiunge che "è vero, come ha affermato Wieland, che Aristotele muove da un'alaisi del linguaggio comune e per "causa" intende anzitutto dei discorsi, cioè le diverse possibili risposte alla domanda "perché?", ovvero delle funzioni concettuali, cioè dei "concetti della riflessione" Ma è altrettanto vero che, per mezzo di discorsi, o di tali concetti, egli allude a "cose". Cf. BERTI, E. Nuovi studi aristotelici. II - Fisica, antropologia e metafisica. Brescia: Morcelliana, 2005, p. 44.

${ }^{20}$ Cf. MCDOWELL, J. Mind and World. Cambridge: Harvard University Press, 1994, p. 78s. Sullo stesso tema cf. SGARBI, M. I fondamenti aristotelici della filosofia della mente di John McDowell. Fenomenologia e società, 39, p. 52-68 (2006); BERTI, E. Aristotele nel Novecento. Roma-Bari: Laterza, 2008, p. v-xxii.

${ }^{21}$ ARISTOTELE, Ethica Nicomachea, VI.3, 1139 b 16-17.

${ }^{22}$ ARISTOTELE, Ethica Nicomachea, VI.3, 1139 b 33-34.

${ }^{23}$ ARISTOTELE, Ethica Nicomachea, VI.6, 1141 a 6-8.

${ }^{24}$ ARISTOTELE, Analytica posteriora, II.19, $99 \mathrm{~b} 35$. 
I rapporti fra scienza e abito dei principi vengono sviluppati ulteriormente da Aristotele nel capitolo conclusivo del secondo libro degli Analytica posteriora. In Analytica posteriora II.19 Aristotele descrive l'acquisizione dell'abito dell'intelligenza. Esiste per Aristotele una capacità innata di discernere (dynamis symphutos kritike) che si chiama sensazione (aisthesis) ${ }^{25}$. La sensazione appartiene a tutti gli animali e non ha bisogno di essere sviluppata, essa è già connaturata nella loro anima ${ }^{26}$. Essa però determina ciò può essere conosciuto da ciò che non lo può essere, cioè implica già un'attività che Aristotele sembrava negare nei libri del De anima. In alcuni animali la sensazione produce una permanenza del sensibile e in altri invece no ${ }^{27}$. Negli animali nei quali permane il sensibile si produce, dopo molti episodi siffatti, la memoria e da questa l'esperienza ${ }^{28}$. Dall'esperienza si forma in aggiunta una nozione universale dei casi particolari ${ }^{29}$. Attraverso questo processo si acquisisce l'habitus della conoscenza scientifica che permette l'intellezione dei principi ${ }^{30}$. Sia il nous che l'episteme sono perciò due abiti, cioè due elementi discorsivi e non intuitivi della conoscenza che dipendono dall'acquisizione di esperienze pregresse fatte dal soggetto.

In questo concetto discorsivo di ritenzione del sensibile e di formazione prima dell'immagine e poi dell'universale (katholou) emerge la riflessione. La riflessione, infatti, è quell'atto logico che permette di interiorizzare la cosa conosciuta e farne oggetto della conoscenza. Tale interiorizzazione è seguita dal fatto che l'universale stesso prodotto dalla mente è esso stesso soggetto a riflessione. Infatti, avendo colui che conosce come cosa conosciuta un suo prodotto, cioè l'universale, il conoscente non può comprendere il particolare se non riflettendo su se stesso e considerando ciò da cui astrae l'universale:

[...] l'intelletto (nous) è esso stesso intelligibile (noetos) come lo è il pensato ( $t a$ noeta). Così nel caso degli oggetti senza materia, colui che pensa (to nooun) e il pensato (to nooumenon) sono la stessa cosa. Perciò la scienza teoretica (episteme theoretike) e ciò che si conosce scientificamente (episteton) s'identificano ${ }^{30}$.

\footnotetext{
${ }^{25}$ Ibidem.

${ }^{26}$ ARISTOTELE, Analytica posteriora, II.19, 99 b 36-37.

${ }^{27}$ ARISTOTELE, Analytica posteriora, II.19, 100 a 4.

${ }^{28}$ ARISTOTELE, Analytica posteriora, II.19, 100 a 2.

${ }^{29}$ Sul rapporto fra hexis e epagoge in Analytica posteriora II.19 cf. KAHN, C.H. The Role of Nous in the Cognition of Frist Principles in Posterior Analytics II 19. In: BERTI, E (cur.). Aristotele on Science. The "Posterior Analytics". PROCEEDINGS OF THE EIGHTH SYMPOSIUM ARISTOTELICUM HELD IN PADUA FROM SEPTEMBER, 7 to 15, 1978, Padova: Antenore, 1981, p. 385-414.
}

${ }^{30}$ ARISTOTELE, De anima, II.4, 430 a 2-6. 
Questa attività per Aristotele non rappresenta altro che quello stato dell'anima in cui si scoprono le condizione soggettive che rendono possibile non solo la conoscenza dei concetti, ma anche attraverso di essi il dato particolare dell'esperienza.

Tutto ciò però non sarebbe possibile se colui che conosce non avesse un proprio specifico habitus che designa in modo particolare l'egoità del conoscente, cioè la costruzione di un centro al quale si possono riferire e sedimentare le diverse esperienze. L'abito designa una "seconda natura": “[...] l'abitudine (ethos) è in un qualche modo simile alla natura, poiché "spesso" e "sempre" sono vicini; la natura è di ciò che è sempre, l'abitudine di ciò che è spesso" 31 . L'abito (hexis), infatti, differisce per Aristotele dalla disposizione (diathesis) per il fatto di essere più stabile e duraturo, e quindi più difficilmente rimovibile ${ }^{32}$. L'abito è quindi qualcosa che compete essenzialmente al soggetto una volta che esso viene acquisito, cioè rappresenta l'unico filtro attraverso il quale è possibile guadagnare una conoscenza di tipo scientifico. Riflessione ed egoità risultano essere perciò due caratteristiche inscindibili ed essenziali senza le quali non sarebbe possibile avere alcuna conoscenza.

Nel presente saggio si è voluto evidenziare che nella teoria aristotelica della conoscenza è implicita una proto-teoria della soggettività grazie alla presenza di elementi quali il punto di vista, la determinazione, la significatività, la riflessione e l'egoità. Questa teoria aristotelica, piuttosto che quella platonica, sta a fondamento della svolta epistemologica del pensiero moderno attraverso le elaborazioni concettuali che l'aristotelismo rinascimentale ha fornito ${ }^{33}$. È forse questo il motivo dei grandi elogi hegeliani ad Aristotele nella prima grande ricerca filosofica volta a dimostrare la genesi della soggettività, cioè la Philosophie des subjektiven Geistes:

[...] i libri di Aristotele sull'anima, con i suoi trattati sui diversi aspetti e stati particolari di questa, sono pur sempre la migliore opera d'interesse speculativo, per non dire l'unica, su questo argomento [...] Lo scopo essenziale di una filosofia dello spirito - prosegue Hegel - può essere soltanto quello d'introdurre di nuovo nella conoscenza dello spirito il concetto, risvegliando così anche la comprensione del senso di quei libri aristotelici ${ }^{34}$.

\footnotetext{
31 ARISTOTELE, Rhetorica, I.11, 1370 a 7.

32 ARISTOTELE, Categoriae, I.8, 8 b 27-28.

33 Sul problema della soggettività nell'aristotelismo rinascimentale cf. Pozzo, R. Ramus and Other Renaissance Philosophers on Subjectivity. Topoi, 22, p. 5-13 2003.

${ }^{34}$ HEGEL, G.W.F. Gesammelte Werke. Hamburg: Felix Meiner Verlag, 1992, X, p. 390.
} 
È quello che si è tentato di fare in questa indagine analizzando il problema della soggettività in Aristotele: far rivivere lo spirito essenziale della sua filosofia e interrogarlo nella sua classicità.

SGARBI, Marco. Aristotle on Subjectivity. Trans/Form/Ação, Marília, v. 34, n.3, p. 105116, 2011

ABSTRACT: Philosophers usually attribute to modernity the invention of concepts like 'subject', 'object' and 'subjectivity' and they understand them in contrast to Scholastic and Aristotelian philosophies. The present paper suggests the presence of a proto-theory of subjectivity in Aristotle. The Aristotelian theory of subjectivity is grounded principally on four doctrines. First, we can recognize the presence of a 'perspective' and of a 'point of view' in the Aristotelian epistemology. The second relevant aspect is the concept of determination of the object of knowledge, which is in Aristotelian philosophy the notion of 'prosthesis'. The third doctrine lies on the distinction between 'what is more known to us' (gnorimoteron emin) and 'what is more known by nature' (gnorimoteron te physei). The last doctrine concerns the 'second nature' of the subject and the habits of knowledge acquired by experience, which explain why knowing subjects have different cognitions of the same object according to their own previous experience.

KEYWORDS: Aristotle. Subjectivity. Determination. Perspective.

recebido em: 01.08.2011

aprovado em: 03.10.2011 